\title{
THE THIERSCH GRAFT. ITS PREPARATION AND USES.
}

\author{
By T. POMFRET KILNER, F.R.C.S.,
}

Plastic Surgeon, St. Andrew's Hospital, Dollis Hill; London County Council; Queen Mary's Hospital, Roehampton; Princess Elizabeth of York Hospital for Children, Shadwell; and Lord Mayor Treloar Hospital, Alton.

No further excuse is offered for the publication of this brief paper than the writer's conviction that just as it is essential for the man practising plastic surgery as a specialty to have a sound practical knowledge of general surgery, so a working knowledge of plastic surgical procedures should be acquired by the general surgeon.

It will probably be many years before hospitals in this country will see the call for special departments for plastic (reconstructive) cases, and it will be longer still before hospitals recognise the necessity for setting aside beds for this special type of work. Until that time arrives, the general surgeon should be prepared to give his patients the benefit of the great advances which have been made in reconstructive work since the European War: otherwise cases will have to go untreated for long periods, living despondent lives on the long waiting lists of those few Centres devoted to this work and producing, in the meantime, secondary deformities or complications, increasingly difficult of treatment: or they will become outcasts from ordinary social life, incapable of obtaining employment and miserable in the extreme.

The general surgeon should be prepared to cover any raw surface he produces: he should be prepared to carry out destructive operations, such as those for the eradication of malignant disease, unfettered by a fear of leaving disfigurement and confident that his operation can be planned in a manner calculated to avoid permanent deformity: he should be prepared to prevent those extreme contractures which so frequently come into the plastic surgeon's hands for correction: he should be prepared to treat such conditions as ectropion before, and not after, loss of eyelid protection has resulted in corneal ulceration and opacity. For all these requirements he should know in more practical fashion than is usually the case how to make use of free skin grafts and simple flaps.

This paper will deal with only one form of free skin graft, the Thiersch or Epidermal graft.

\section{Nature of the Thiersch or Epidermal Graft.}

The average conception of a Thiersch graft is a fragment of skin little larger than a postage stamp, and frequently a look of something akin to awe has been seen in the eyes of a surgeon watching, for the first time, the cutting of a Thiersch graft of, say, three by six inches. Now, this should not be so, for it requires only a reasonable degree of skill and a limited amount of patience and practice to cut grafts of the full size of any available skin surface.

It must be remembered clearly that a Thiersch graft consists of only the surface epithelial layers. When cut, it should be uniform in thickness and should have the appearance of thin tissue paper. It should leave a surface, with only tiny 
THE THIERSCH GRAFT. ITS PREPARATIONS AND USES.

FIG. 1.

(a) The upper arm properly draped in preparation for the cutting of a graft.

(b) The arm supported on the surgeon's knee.

The wood slip and knife in position for cutting the graft.

(c) The thigh suitably draped and placed in correct position for graft cutting. The wood slip and knife are in position for cutting from the broadest skin surface available.

(d) Wood slip and knife in position for graft cutting in the proximo. distal direction recommended.

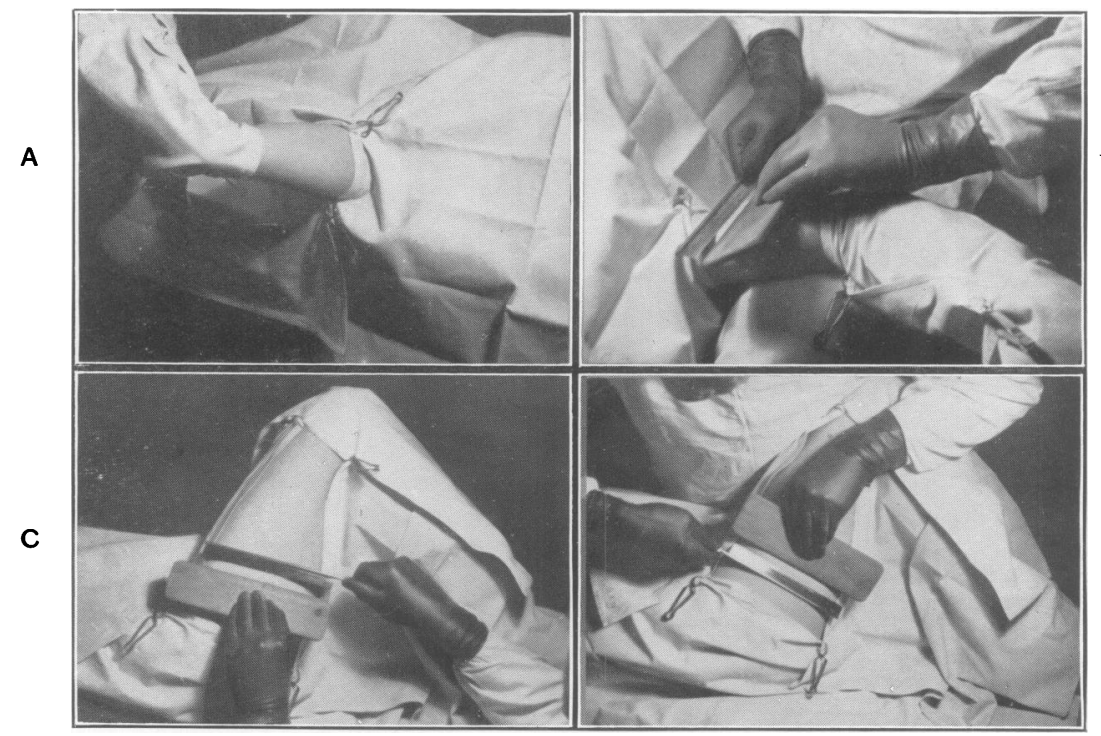

FIG. 1.

FIG. 2

Congenital Syndactyly treated by Thiersch grafting of reconstructed interdigital clefts. 


\section{THE THIERSCH GRAFT. ITS PREPARATIONS AND}

USES.

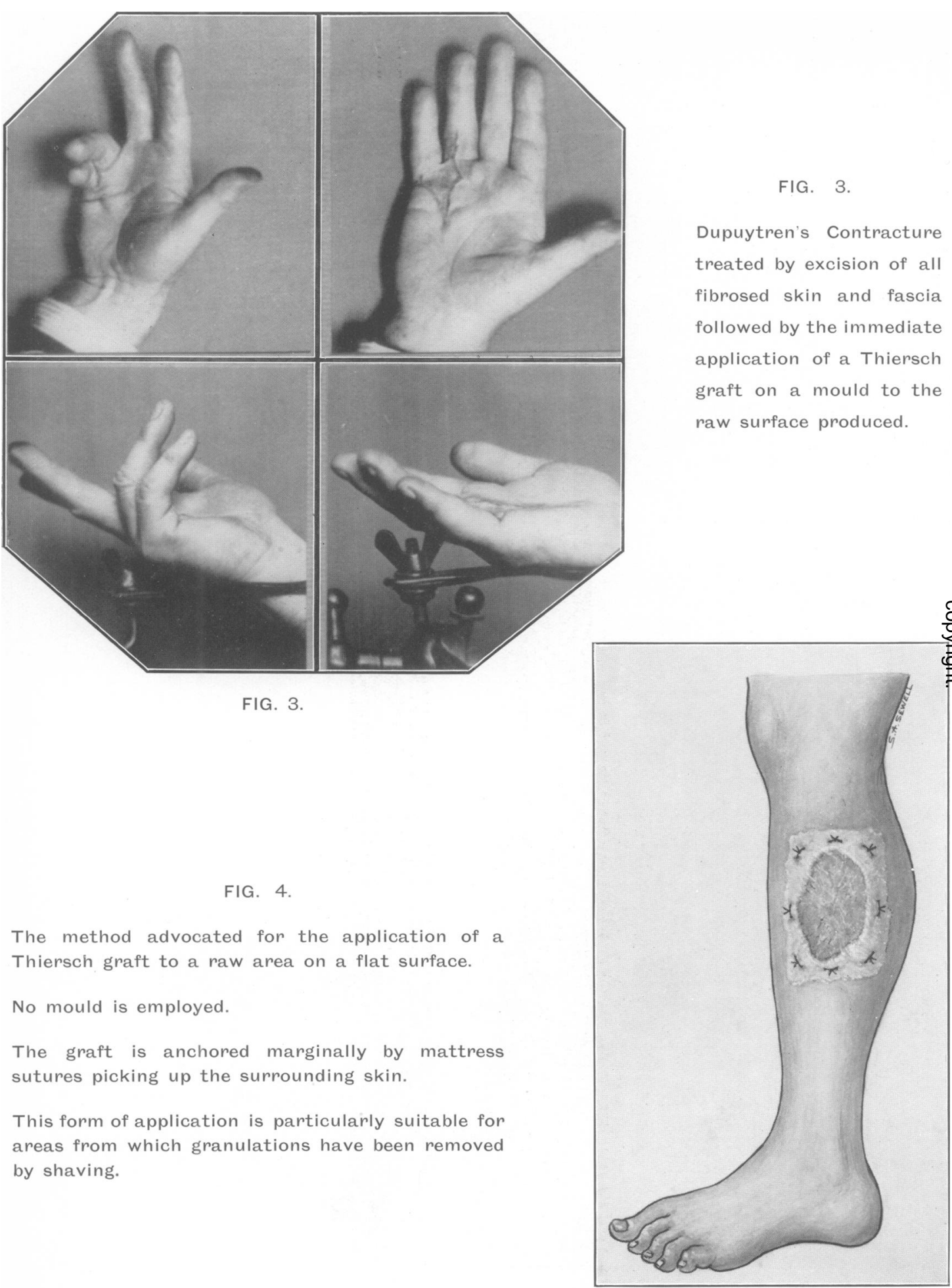

FIG. 4 . 
FIG. 5.

Extensive burns of the buttocks and thighs treated by shaving of granulations and application of marginally anchored Thiersch grafts.

The white spotted areas on the left thigh were particularly refractory and were treated by pinch graftssmall deep grafts with Thiersch graft fringes; the site from which they were removed is seen on the outer aspect of the right thigh.

In this case the burnt areas had remained unhealed for 3 months.

After grafting no contracture was present.

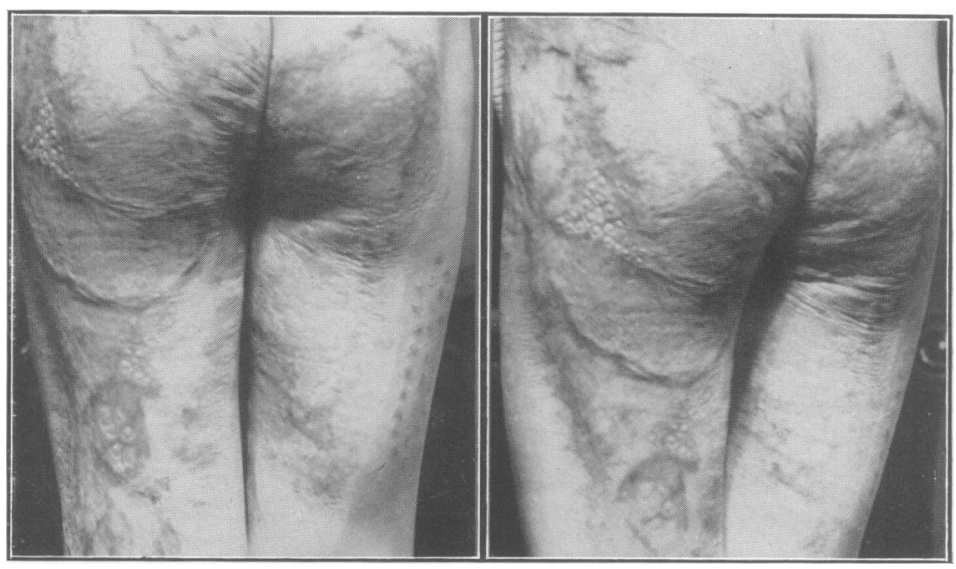

FIG. 5 .

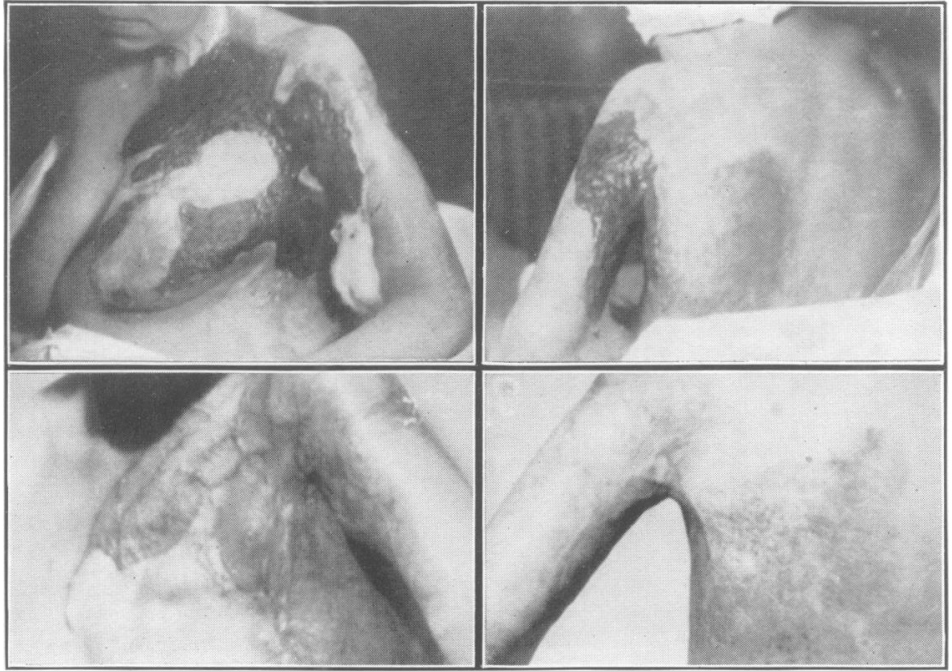

FIG. 6.

Case of severe burns of chest wall, back, and arm unhealed after 19 months treatment elsewhere.

Satisfactory healing, with only slight limitation of arm movements, obtained by multiple Thiersch grafts.

FIG. 6. 
punctate hæmorrhages, on which epithelial growth is so rapid that healing is complete when the dressing is removed for the first time at the end of a week. It is in this respect that the Thiersch graft is so surgically economical; the donor area suffers little and may even be used again as the source of a graft at some later date. The unpleasant keloid scars frequently encountered on patients' limbs, stated to be the sites from which Thiersch grafts have been taken, are evidence that many surgeons do not understand clearly what a Thiersch graft is, for it is obvious that the knife has penetrated deeply at some points or throughout the graft.

In the majority of cases, Thiersch grafts are required to be hairless and they are, therefore, usually taken from the inner aspect of the upper arm or the outer side of the thigh. The antero-internal aspect of the thigh is a difficult region from which to cut a good graft of uniform thickness and breadth for it is impossible to place the limb in any position which does not leave the adductor longus producing an unpleasant and greatly-interfering ridge. Unless it is immaterial whether the graft, in its transplanted position, does or does not grow hair, it is unwise to have the donor area shaved beforehand for it then becomes difficult to decide what is and what is not hair-bearing skin. These remarks are made in spite of the fact that the writer is aware that, in theory at any rate, no Thiersch graft should carry with it hair follicles and, therefore, the capacity for hair growth. It is always well to be on the safe side and avoid the necessity of removing, for example, those intra-oral beards which have more than once been seen following skingrafting in the mouth.

\section{Preparations for removal of Graft.}

Preliminary skin preparation should be carried out without pigmented antiseptics and ordinary soap-and-water cleansing followed by methylated ether and then by alcohol has proved best for the purpose. At the time of actual operation a final wipe over with ether is all that is required: methylated spirit is inclined to make the knife slide less smoothly over the surface. When it is known beforehand roughly what area is to be grafted, it is as well to cut the graft first (and to cut it well over-size) in order to avoid the possible carrying of infection from the recipient area to the innocent, sterile donor area. Draping of the area should not be carried out in any slip-shod fashion for it should be considered "criminal" to infect the area from which the graft is cut.

At the risk of appearing to give unnecessary detail, explicit instructions regarding preparation may be given, for there is no doubt that many a graft is doomed to failure from infection before it reaches its destination.

Experience has shewn that there is only one completely satisfactory way of shutting off the arm area and this is illustrated in Fig. I $a$. The arm is held out from the body horizontally by a nurse for final skin preparation. A sterile towel is placed over the chest, level with the axilla and coming well down the side of the table. The upper arm is cleaned with ether as far as possible in this position. The arm is now raised to a vertical position, the already cleaned part coming into contact, if with anything, with the sterile towel and not with the blanket. The remaining surface of the limb is cleaned with ether and two fresh towels, placed over the chest and face respectively, are clipped to the skin above and below as in the illustration. A ring of mastisol is painted round the elbow region and a third towel is carefully draped over the hand and forearm with its upper edge glued by mastisol. A towel clip is inserted for greater safety. The nurse 
continues to hold the arm with the palm of the hand upwards during the cutting of the graft. She should not pull on the arm but should allow the elbow to be slightly flexed while the upper arm rests on the knee of the surgeon whose foot is supported on a stool of convenient height (Fig. Ib.).

Everything is now ready for the cutting of the graft. A sharp Thiersch knife or a newly stropped razor, and a strip of wood with smooth rounded edges, eight inches by three inches and a quarter-of-an-inch thick, are all that is required for the job. The under surface of the knife or razor, and particularly its back edge, are smeared lightly with vaseline or liquid paraffin and one edge of the wood strip is similarly treated. Too liberal a supply of grease on the wood strip renders the skin surface of the graft unduly greasy and makes its later manipulation unnecessarily difficult.

\section{The Knife.}

It is worth while diverging a little at this point to make some remarks on skin-grafting knives. There is little question that the best knife for the purpose is one of ample length prepared from wafer steel of the pattern made by Tontrup of St. Louis, to the requirements of Professor V. P. Blair. This knife has been copied for the writer in this country by Messrs. Down Bros. and is supplied with a very simple sheath which prevents damage to its edge when not in use. It is quite unnecessary and, indeed, disadvantageous to have such a knife ground and set after every operation. Blair himself employs a damp sterile towel smeared with sterile emery flour for stropping the instrument during the course of an operation, but the ordinary care which a man gives to his "cut-throat" razor for shaving purposes is all that is required to keep a graft-cutting knife in excellent condition for manyo months and for a very large number of operations. The knife should be carefully washed and dried after use and should be stropped immediately; it should be stropped again immediately before use. It is best sterilized in lysol followed by alcohol or in the latter alone. Expensive strops are supplied by most surgical instrument makers but none of these has been found one tithe so useful as a very low-priced three-sided strop, the Rosswaye Tri-side Strop, made and marketed by R. M. Ross, 26, Upton Road, Slough.

The ordinary French razor made of soft steel to which an excellent cutting edge can be given (even after considerable damage has been done to it) by a few strokes on such a strop, is eminently suitable for cutting smaller grafts, only its limited length precluding its use for larger ones. A special metal handle has been made for the French razor blades by Messrs. Thackray, which allows for the locking of the blade in the open position. The older, more expensive and heavier Thiersch graft knife can be kept in good condition in precisely similar manner and at times one has felt that its weight and balance offer certain advantages.

\section{Cutting the Graft.}

The greased edge of the wood slip is applied transversely across the upper arm skin, just below the axilla, and its position is changed until a flat surface sufficiently broad for the required graft is produced. At one time a second wood slip was held above by an assistant but this and all stretching or other manipulation of the skin were discarded many years ago for these "helps" represent unknown factors and success depends upon a nice balance between the work of the two hands of the operator. In this procedure it is very essential that the right hand shall know precisely what the left hand is doing. 
The knife now begins its work. It is applied to the skin surface just proximal to the wood slip and is worked steadily to and fro with a gentle sawing movement until it begins to cut. Just as a saw should be allowed to cut its own way through wood, so no undue force should be employed to make the knife cut through the epithelial layers of the skin. It is difficult to explain exactly what is to be done with the wood slip. Sometimes it is necessary to stretch the skin by drawing it down the arm, sometimes to press the edge firmly against the skin, but usually one finds that only the very lightest contact is required to render the surface sufficiently taut to allow of easy cutting. As the knife advances so the slip recedes before it, sliding easily over the skin by virtue of its greased edge. Practice on a piece of chamois leather stretched over a board is well worth while, for only practice can develop the proper touch.

Given a sharp knife, a modicum of skill and a reasonable amount of patience, graft-cutting should offer no serious difficulty. To this list of requirements, one should perhaps add a good patient, for there certainly are occasional individuals from whose skin it is extremely difficult to cut good grafts. In these it is probably undue thinness of skin which causes the difficulty, for when the skin is very thin and the tension produced by the wood is too great it is the easiest thing in the world to cut through to fat, the skin splitting as though its contents were under tension and anxious to escape.

There is no need to repeat detailed technique for cutting grafts from the leg. Figure Ic. illustrates the best position of the limb. It is worth noting that it is always easier to cut grafts in a proximo-distal than in the reverse direction. For this reason when cutting from the leg, one frequently leans over the limb, cutting in the way illustrated in Fig. Id.

The graft obtained, it is laid flat on a sheet of gauze moistened with warm saline and is covered by another similar piece of gauze and placed on the table suitably guarded by a red towel or other means, lest it should be lost before it is required. This precaution is necessary, for though it barely seems possible, grafts have been seen reaching the main operation area long before they were really required, dragged there unwittingly in swabs or adhering to the sleeve of an assistant or nurse.

The area from which the graft has been cut is now wiped dry and covered with a layer of Tulle Gras over which are placed several layers of gauze wrung out of saline. Tulle Gras is an open mesh net material impregnated with vaseline containing I per cent. Balsam of Peru. It is prepared by Lumière, of Paris, and is supplied by the Anglo-French Drug Co. It has been found particularly suitable for dressing these areas. Previously, lint, spread or impregnated with vaseline, was employed but this provided a much too air-tight dressing preventing the escape of serous or other discharge and converting the area covered by it into something of a hot-house in which organisms from the glandular elements of the surrounding skin appeared to thrive. Various modifications of the original material and instructions for its preparation for Hospital use are described in an excellent paper by D. A. Beattie, published in the R.A.M.C. Journal, I933, xl., p.352.

After the application of this dressing, the arm is entirely discarded by the surgeon and handed over to a nurse for bandaging, the towels which have already been used being removed. 
The recipient area for the graft is now dealt with as an entirely separate operation. Only in this way is it possible to maintain any degree of asepsis throughout such a two-stage operation. There are, of course, exceptions to this rule, when, for instance, a graft is cut from one part of an arm or leg for transfer to some other part of the same limb. In these cases the whole limb can be prepared at once and there is no need to divide the operation into two such definitely separate procedures.

\section{Application of the graft.}

Whenever the raw surface to be covered possesses cavity form it is advisable to make an accurate mould of it in dental modelling composition (Stent). Such a mould greatly facilitates the application of the graft and ensures contact of the graft at all points on even the most irregular surface.

The tablets of stent are best prepared for use by standing in I/20 carbolic solution until required. This sterilizes their outer surfaces which have been subjected to handling. They may, alternatively, be kept sterilized in a jar containing formalin vapour and are then handled by forceps. When required they are immersed in boiling water and when soft are worked up into a smooth mass which is applied to the raw surface and pressed home to fill the whole defect. It has been found of advantage to stretch over the mould a piece of gauze held in such a manner as will imitate the pressure which will be produced by the final dressing. While so held in position the Stent is hardened by dripping over it a stream of iced saline either from a swab or, more effectively, from a dental chip syringe.

When set, the mould is removed and dried. Careful note is made of the impression area and over this the graft is uniformly spread, raw surface outwards.

When the surface to be covered is very large, several grafts may be required. These should be so arranged that their edges overlap slightly.

The raw surface is cleared of blood clots and the graft-covered mould is applied. The skin surrounding the defect is wiped with ether and is smeared with mastisol, as is also the exposed surface of the mould. The margins of the graft or grafts are turned over on to the surrounding skin edges and the mould is provided with initial fixation by the application of ribbon gauze strips held by the mastisol.

The whole area is covered by layers of gauze or wool and firm pressure is maintained by either crêpe bandage or elastoplast strapping.

Fig. 2 illustrates the result to be expected from grafts applied to the interdigital clefts on moulds in this manner, while Fig. 3 illustrates the result of grafting the slightly depressed area produced by the excision of fibrosed skin and fascia in a case of Dupuytren's contracture. An area commonly treated in similar fashion is the large depressed raw surface left by excision of an X-ray burn of the back.

The writer no longer uses the Stent mould when the raw area is on a flat surface for he feels that in such situations the mould is liable to shift its position and drag the graft from its bed. Figure 4 indicates the technique employed in such areas. The graft is spread on the raw surface, rendered as bloodless as possible by pressure and the application of iced saline, and is marginally sutured by mattress stitches of fine silk-worm gut passed on an eyeless needle. When 
several grafts are required, their edges are slightly overlapped and may be fixed by occasional mattress stitches passing through the two layers of graft and picking up the raw surface below. The graft is covered by a layer of Tulle Gras and any underlying blood is expressed by rolling over the area a folded gauze swab. A pressure dressing of cotton wool soaked in flavine and paraffin emulsion is then built up over the graft and the whole dressing is firmly fixed by either elastoplast strapping or by a crêpe bandage. Careful splinting of a limb so treated must not be overlooked.

When the surface to be grafted is a granulating one, it is advisable to shave off the granulations with a sharp scalpel or graft knife. This removes a layer of tissue which is likely to be infected and which, in any case, produces discharge capable of preventing proper apposition of the graft. It leaves a surface with punctate hæmorrhages very like that left in the donor area. Figs. 5 and 6 illustrate cases treated in this manner.

In the neck region a similar method is employed, though here it is sometimes advisable to use a Stent mould of ample proportions which will act in the dual capacity of pressure dressing and splint to restrict movement of the parts.

Plaster of Paris fixation has a definite place in all plastic work but it has to be remembered that it is difficult to prepare plaster casts beforehand for cases in which contractures are present and their preparation at the time of operation adds a further long period to what has probably already been a rather prolonged operative procedure.

\section{After-Treatment.}

The donor area is left entirely untouched for at least a week, and it is usual at that time to be able to peel off the dressing readily and painlessly and to find a completely epithelialised surface already present.

The grafted area is also left undisturbed for 7 to Io days unless rise of temperature, pain or excessive discharge indicate the need for earlier dressing.

The outer dressings are removed down to the mould. The edges of this, which have become glued by inspissated serum and blood, are moistened with peroxide of hydrogen until the mould can be loosened and removed without dragging the newly adherent graft with it.

Redundant edges are trimmed away and the surface of the graft is gently dabbed clean of secretions. Any blisters which have formed owing to collections of blood or serum under the graft are opened and evacuated.

Pressure is maintained by bandage over cotton wool or gauze soaked in flavine and paraffin emulsion. The dressing is changed every 2 to 3 days and a fortnight after operation the grafted area should be ready for gentle grease massage. Liquid paraffin, lanoline, or cocoa butter may be employed for this purpose. Drying up of the graft should be prevented by keeping its surface always slightly greasy. The after treatment where no mould has been employed is precisely similar but frequently there is no need to disturb the layer of Tulle Gras at the first dressing.

The application of the Thiersch graft to special areas will be dealt with in a subsequent article. 